\title{
QUEEN'S
UNIVERSITY
BELFAST
}

\section{A Cost Analysis of Machine Learning Using Dynamic Runtime Opcodes for Malware Detection}

Carlin, D., O'Kane, P., \& Sezer, S. (2019). A Cost Analysis of Machine Learning Using Dynamic Runtime Opcodes for Malware Detection. Computers \& Security, 85, 138-155. https://doi.org/10.1016/j.cose.2019.04.018

\author{
Published in: \\ Computers \& Security
}

Document Version:

Peer reviewed version

Queen's University Belfast - Research Portal:

Link to publication record in Queen's University Belfast Research Portal

\section{Publisher rights}

Copyright 2019 Elsevier.

This manuscript is distributed under a Creative Commons Attribution-NonCommercial-NoDerivs License

(https://creativecommons.org/licenses/by-nc-nd/4.0/), which permits distribution and reproduction for non-commercial purposes, provided the author and source are cited

\section{General rights}

Copyright for the publications made accessible via the Queen's University Belfast Research Portal is retained by the author(s) and / or other copyright owners and it is a condition of accessing these publications that users recognise and abide by the legal requirements associated with these rights.

Take down policy

The Research Portal is Queen's institutional repository that provides access to Queen's research output. Every effort has been made to ensure that content in the Research Portal does not infringe any person's rights, or applicable UK laws. If you discover content in the Research Portal that you believe breaches copyright or violates any law, please contact openaccess@qub.ac.uk. 


\title{
A Cost Analysis of Machine Learning Using Dynamic Runtime Opcodes for Malware Detection
}

\author{
Domhnall Carlin*,Philip O’Kane ${ }^{\dagger}$, Sakir Sezer ${ }^{\star}$ \\ Centre for Secure Information Technologies, Queen's University, Belfast, Northern Ireland \\ Email: *Corresponding author: dcarlin05@qub.ac.uk, ${ }^{\dagger}$ p.okane@qub.ac.uk, ${ }^{\ddagger}$ s.sezer@qub.ac.uk
}

\begin{abstract}
The ongoing battle between malware distributors and those seeking to prevent the onslaught of malicious code has, so far, favored the former. Anti-virus methods are faltering with the rapid evolution and distribution of new malware, with obfuscation and detection evasion techniques exacerbating the issue. Recent research has monitored low-level opcodes to detect malware. Such dynamic analysis reveals the code at runtime, allowing the true behaviour to be examined. While previous research uses machine learning techniques to accurately detect malware using dynamic runtime opcodes, underpinning datasets have been poorly sampled and inadequate in size. Further, the datasets are always fixed size and no attempt, to our knowledge, has been made to examine the cost of retraining malware classification models on datasets which grow continually. In the literature, researchers discuss the explosion of malware, yet opcode analyses have used fixed-size datasets, with no deference to how this model will cope with retraining on escalating datasets. The research presented here examines this problem, and makes several novel contributions to the current body of knowledge.

First, the performance of 23 machine learning algorithms are investigated with respect to the largest run trace dataset in the literature. Second, following an extensive hyperparameter selection process, the performance of each classifier is compared, on both accuracy and computational costs (CPU time). Lastly, the cost of retraining and testing updatable and non-updatable classifiers, both parallelized and non-parallelized, is examined with simulated escalating datasets. This provides insight into how implemented malware classifiers would perform, given simulated dataset escalation. We find that parallelized RandomForest, using 4 cores, provides the optimal performance, with high accuracy and low training and testing times.
\end{abstract}

This work was supported by EPSRC under Grant CSIT 2 EP/N508664/1. The funder had no involvement in the study design; in the collection, analysis and interpretation of data; in the writing of the report; and in the decision to submit the article for publication.

We would like to thank the reviewers and editorial team for their constructive reviews of the initial drafts of this paper.

Index Terms-Malicious code, network security, machine learning, computer security, malware.

\section{INTRODUCTION}

From the earliest PC viruses in the 1980s, to highly sophisticated modern malware (malicious software), cyber-threats to individuals, companies, public bodies and even nation states have escalated at an alarming rate. The aim of the ongoing fight against malware is the ability to detect, prevent and mitigate deliberately malicious software before damage can occur. However, current anti-virus (AV) solutions are failing to detect the latest highly-evolved malware.
The most common approach to malware detection within commercial AV products is signature detection [1]. Suspect files are scanned for pre-learned signatures (strings, code segments, URLs etc) stored within the product database. By necessity, new malware instances must be captured, analysed and a signature derived, which updates the database for all users. By definition therefore, this approach is behind the curve when new malware is released. The lag between the release of the malware in the wild and the release of signatures to users can prove costly. With the unending evolution of detection evasion techniques, now even offered as a paid-for service on dark market sites, signature detection is struggling to cope with the explosion in malware campaigns.

Recent research into strategies for detecting obfuscated malware have used dynamic analyses to allow the true code to be revealed at runtime. Specifically, dynamic opcode analysis investigates the assembly language instructions passed to the CPU at runtime (opcodes). Operational codes are the humanreadable portion of the assembly code, used to perform various operations on the CPU to which the instruction set belongs. With such a low-level focus, this approach allows the direct investigation of malware behaviour, including any attempts at anti-analysis techniques. Machine learning has been applied to the runtraces yielded by these analyses, with the aim of building a predictive model which is immune to obfuscation and capable of detecting unseen malware.

With new instances of malware estimated at around 31 million in Q1 2017 (345,000 per day) [2], machine learning techniques must evolve in order to maintain adequacy in the detection of malicious software. Domingos and Hulten [3] state that knowledge discovery systems are limited by three factors: time, memory and sample size. Traditionally sample size is the key issue, which has been discussed at length in [4] in the context of malware analysis, particularly dynamic analyses. Limited sampling provides increased risk of overfitting a model to an undersized dataset. However, with the increase in sample sizes to more accurately model the current threatscape, the risk moves to under-fitting. This is where the limitations of memory and time constrain the available processing and algorithms, rather than low sample sizes. The result is under-utilisation of the data, where all the instances cannot be processed and incorporated as training samples, giving an overly-simple model. This is an actively-researched area within data mining and machine learning as, with the exponential growth in information to mine, capable algorithms are key to taking full advantage of such enormous volumes 
of data. With the recent explosion in malware distribution, the limitations discussed should be a problem for machine learning approaches to dynamic analyses. However, it currently is not, which underlines the inadequacy of the datasets underpinning research to date.

The remainder of this paper is presented as follows. Section II presents a brief synopsis of previous research on dynamic opcode analysis along with investigations into incremental and non-incremental learning algorithms and their applications to malware analysis. Section III illustrates the methodology used to generate and conduct our analyses, the results of which are presented in section IV. Finally, section V discusses these analyses and presents our conclusions.

\section{RELATED WORK}

\section{A. Dynamic opcode analysis}

Dynamic opcode analysis has been investigated successfully in the literature. Santos et al [5] investigated small sequences (n-grams) of opcodes in statically yielded opcode representations. Their machine learning model (Support Vector Machine) achieved accuracy rates of $>85 \%$ when distinguishing from benign ware. Runwhal, Low and Stamp [6] used graph algorithms to the opcodes from metamorphic malware, extending the work of [7]. Their model was capable of distinguishing metamorphic malware from benign executables and also metamorphic malware from other malware types. O'Kane et al [8] dynamically investigated opcodes in the detection of malware using supervised machine learning. Run-time traces of benign and malicious Windows Portable Executables (PE) were taken using a virtual machine to execute the file. Virtualization allowed malicious files to be investigated in a controlled environment, operating on an additional layer above the host machine's operating system. Carlin et al. [9] used dynamic opcode analysis to classify almost 48,000 malicious PEs, while examining the impacts of run-length (number of opcodes recorded) and n-grams. The authors reported that $32 \mathrm{k}-$ length opcode traces, using $n=1$ (i.e. no sequences) offered the highest accuracy $(99.05 \%)$ with a Random Forest classifier and using 10-fold cross-validation. This indicates dynamic opcode analysis can quickly detect the malicious behaviours of unseen malware with high levels of accuracy.

\section{B. Incremental and non-incremental learning}

Non-incremental classifiers build a model once, based on the whole set of instances provided at training time. An incremental learning method (also referred to as updatable, online, sequential or successive methods[3]) creates a base model from the initial training instances presented to it, but can subsequently revise and evolve the concept model based on new instances it encounters. Algorithms capable of online learning only learn incrementally- considering each training instance once, then disregarding it; batch-based learners have access to all instances in memory [10]. Utgoff [11] notes that non-incremental learners are appropriate when a fixed set of training data is presented, but incremental classifiers are appropriate when the training instances are in a stream format. This would cause one to question how a stream of atomic instances would present, in this case processed malware samples. The problem at hand can be viewed as a serial learning case, where models are repeatedly rebuilt. In this case, [11] suggests incremental learning is preferable, as revising a hypothesis continually may be much more efficient than continually building a new one. [12] stated that the cost of updating incrementally will be lower on average than rebuilding a tree, although individual updates may be more expensive. However, updatable learners can require more data than the non-updatable version, prior to building an acceptable model. They can also be susceptible to negative effects of noise, missing data, over-fitting, order of input data and outdated concepts (i.e. older data reducing the accuracy of a tree)[10].

In [11] (updated in [12]) a new algorithm for incrementally training decision trees is proposed (ID5R). The author examined the cumulative CPU time for training with up to 30,000 instances, when comparing eight variants of the ID3 classifier (ID3', ID5R, ID5, ID4). This included cross-comparing versions of these for classifiers with an alternative training strategy, whereby trees are only altered by new instances should the base model have misclassified the new instance. CPU time was only viewed as a secondary metric, with the main focus being on algorithmic complexity measures: instances used in tree construction, E-score computations, and Node count. The concept of runtime differs for online learners. While the update time can be short, there can often be a need to have more instances in order to build an optimal tree. Further, the work of [12] shows that updating time increases with training, i.e. with the volume of instances. In a survey paper of online decision tree learning algorithms [10], Rosset notes that no amortized analysis had been employed in the literature. Amortized analysis is a worst-case analysis of the overall cost of sequence of operations rather than separately analysing each operation in that sequence.

The most closely related work to [11] is [3], who proposed a VFDT (Very Fast Decision Tree), based on Hoeffding Trees, which learns in constant memory and time per example. The authors criticise the ID5R algorithm as being even slower than its predecessor ID3 on noise-free simple problems, with the expectation that noisy streaming data would only exacerbate this.

Crucially, VFDT offers strong guarantees of asymptotic similarity to the accuracy of corresponding batch-based trees, given enough instances. While accuracy of a batch classifier can be higher when $n$ is small, given enough samples VFDT will outperform non-incremental algorithms [10]. [3] examined this, comparing VFDT to $\mathrm{C} 4.5$ (batch classifier). $\mathrm{C} 4.5$ was more accurate up to 25,000 samples, and similarly as accurate between 25-100,000 samples. However, C4.5 was unable to continue after 100,000 samples, as a memory threshold had been breached (40MB). This was a limit artificially imposed by the authors, being the memory which 100,000 samples would consume, i.e. 100,000 samples was an artificial ceiling for C4.5. As VFDT could employ samples beyond that of C4.5, its accuracy increased at this level, beyond that at which $\mathrm{C} 4.5$ terminated. C4.5 was around $24 \%$ faster to train, although subsequent experiments found VFDT faster to train on 1.61 million samples than $\mathrm{C} 4.5$ did on 75,000. 
Lee and Jun investigated the computational overhead for an updated version of a logistic model tree (LMT) [13]. LMT complements logistic regression with tree induction to harness the advantages of both algorithms. As LMT can harness additive modelling as a form of incremental learning, it is seen as a robust and accurate algorithm. However, the authors note that computational cost associated with LMT prevents it from being adopted in practice. The authors compared their updated algorithm with the original LMT across 14 standard machine learning datasets, measuring training times and accuracy. It was found that there was no statistically significant difference between accuracies of the two distributions using corrected resampled $t$-tests, i.e. the accuracies were comparable. However, it was also found that the training time (seconds) was greatly reduced, with an average of $9 \%$ of the training time of the old algorithm needed by the new algorithm to generate the model.

Sethi and Kantardzic [14] presented the idea of adversarial drift, a malicious subset of concept drift, which causes the distribution of the modelling data to change with time. This differs from standard concept drift, which can be handled with retraining, in that the adversarial activity is designed to purposely degrade the models. This can be maliciously instigated, with an overall aim of avoiding detection with the domain of the classifier. This is particularly relevant to malware analysis using machine learning approaches, as models can be deliberately attacked in order to usher malicious executables past detection systems.

\section{Incremental Learning in Malware detection}

Feng and Han [15] examined the use of a new incremental associative classification algorithm (EIAC) for malware detection using Win32 API calls. A dataset of 10,000 malicious and 10,000 benign PE files was used, with a further 5,000 of each class for a test set. The training samples were split into 5 parts: (1) 12,000 (50/50), (2) 2,000 malware, (3) 2,000 malware, (4) 2,000 benign, (5) 2,000 benign. No explanation is given as to why stratified folds were not used, and why single classes were used for 4 of the 6 retraining sets. CPUtime was recorded, though no definition was given as to how this was measured. When comparing a re-learning algorithm (non-incremental) to the authors' proposed incremental learner, the CPU times were listed as (hrs) 1, 2, 5, fail, fail (i.e. could not complete) and $1,0.5,0.45,0.6,0.65$ respectively. The authors explain this as proof of efficiency of their algorithm for malware classifier updating. The authors then compared their updatable algorithm against two classifiers (Decision Tree and SVM), but only cited the accuracy measures (DT: $89.63 \%$, SVM: $92.58 \%$, EIAC: 93.5\%). No metrics were given regarding retraining times, but the authors stated that the Decision Tree 'exhaust (sic) too much RAM', and any attempt to decrease this rendered the accuracy unacceptable.

Rieck et al [16] used a simulated application, based on their behavioural analysis of API calls rendered as instruction sets, to investigate what the authors termed 'incremental analyses'. This definition differs from incremental learners, as previously described, in that the authors describe a continual batchprocessing approach to update their clusters and classifiers.
The process rendered malware behaviour into a bespoke encoded representation, not dissimilar to opcode/operand notation. Using 33,698 samples, the authors created seven simulated days of data to update their model. They did not, however, seek to discriminate between malicious and benign files, merely to categorise malicious files into either an accepted category, or create a new clustering of similarlybehaved executables. Using this incremental approach, the authors reported large reductions in processing time, with a speed-up factor of 4 for the total process.

Huynh, Ng and Ariyapala [17] investigated an adaptive learning algorithm which was implemented to apply online learning to malware detection. 100k executable samples were obtained from VirusShare, with the cohort used described by the authors as being collected from March 2016 to April 2016. However, they state that from November 2010 to July 2014 was the 'period of most concentration of executables and chosen for the study', which makes the setting unclear, as they rely on compilation time as an indicator of 'in-the-wild' time. The $52 \mathrm{AV}$ scanner reports from VirusTotal were used to designate a file as being malicious or not, but no benign set was used. Samples were then executed virtually using Cuckoo for one minute. This is a questionable design decision, as one minute run-time in dynamic execution would be much shorter than any paper previously reviewed in the present work. No rationale is given for this run-time, nor is any anti-anti-virtualization strategy discussed. 482 features were extracted, categorised into API calls, Registry, File System, and Miscellaneous. Samples were divided into separate datasets based on their month of compilation, averaging 2400 per month. Evaluations of the algorithms were carried out on two separate platforms; Logistic Regression with loss function using TensorFlow and the two online learners using Python with Numpy modules. This is a critical flaw in the methodology, as the authors attempted then to report training and testing times. Being heavily influenced by their implementation, comparing algorithms on the times reported would provide uninterpretable results. The key measurement of success for the algorithms was the mean cumulative absolute error, i.e. digression of the predicted 'maliciousness rating' from the actual. The authors do not explain the maliciousness rating, nor how it is calculated, making the results difficult to interpret.

\section{Aims}

From the literature, there has been scant attention paid to the application of machine learning to escalating data, with studies focussing mainly on single static datasets. This offers no insight into how such models would perform under escalating data conditions, both in terms of accuracy and time taken to generate usable models. This work seeks to investigate these issues in the context of dynamic opcode runtraces for the detection of malware.

The main aims of this paper are as follows:

1) Compare relevant classifiers using default hyperparameters on the novel dataset.

2) Select hyper-parameters using 10-fold cross validation.

3) Evaluate the best versions of each classifier within the current problem. 
4) Examine the cost of training and testing each classifier, beyond the normal accuracy metrics.

5) Simulate escalating data across five 'days'.

6) Examine the cost of training, retraining and testing nonupdatable classifiers.

7) Examine the cost of training, updating and testing updatable classifiers.

As the present paper seeks to evaluate the performance of classifiers on an applied problem, rather than the individual algorithms, we chose to focus on CPU time-based metrics rather than algorithmic time complexity.

\section{Methodology}

The methods used to create the runtime opcode traces and the dataset used here are presented in [4]. For brevity, this process is briefly restated here.

Research in the area has ordinarily used online malware repositories such as VxHeaven [18] or VirusShare [19]. The former is outdated, with the last updates recorded as being in 2010. VirusShare, however, contains substantially more samples, is continuously updated, and includes useful metadata. The three most current folders of malware, at the time of creation, were downloaded and a majority-rules decision was used to filter down the corpus into executable malware i.e. more than $50 \%$ of the $\mathrm{AV}$ scanners detected the file as malicious. The datasets in [20] were also acquired and incorporated, resulting in a total malicious dataset of 43,530 PE files. Benign software was extracted from Windows 7 machines, representing typical PEs which would be encountered in a benchmark environment. The SMOTE algorithm [21] was implemented to oversample the minority class, resulting in 3,591 benign files. Each file was executed within a clean snapshot of a VirtualBox VM running Windows 10 for 9 minutes, providing a balance between execution time and feasibility of dataset creation. OllyDbg V2 was employed to debug each file and provide the run-time trace (i.e. the dynamic opcodes as they were issued to the CPU).StrongOD v0.4.8.892 was used to hide the debugger, as per [8]. The host OS was crafted to simulate a real system e.g. a full recycling bin, browser history, documents history, Flash, Java, .Net installed etc. Anti-virtualization techniques were not utilized in the execution stage. The presence of VBoxGuestAdditions is a key indicator of the system being virtualized, but is necessary to provide guest control over execution and automation, and further mitigation attempts would falter at this point. The aim of dynamic investigations of malware is to experience the executable as a user would normally experience it, including virtualized platforms. Lastly, the anti-virtualization attacks of malware may also provide useful features for detection when operating as low as the opcode level. The run-traces were collected and parsed to count the occurrence of each of the 610 opcodes in the Intelx86/x64 architecture [22]. This formed the feature vector (i.e. each opcode became an individual feature), which was consistent for all classifiers in all experiments. This is more fine-grained than [8] and [23], as past research [24] and [25] has indicated that rarely occurring opcodes may boost malware detection rates. Our past work has indicated that

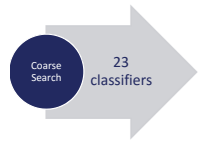

$10 \mathrm{CV}$

10 iterations

Default Params

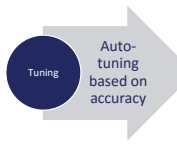

$10 f \mathrm{CV}$ used

to select all

hyperparams

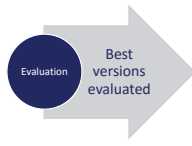

$10 f \mathrm{CV}$ used

Models

retrained and retested on 6 partitions
Fig. 1: Experimental process

feature engineering was not necessary for the current problem, using this same dataset [9]. It is computationally expensive, can increase overfitting and decrease accuracy.

\section{A. Experiment Design}

The experiment was designed to span four phases as depicted in Fig.1:

1) Phase 1: Coarse Search: In the first phase of the experiment, a coarse search of algorithms was performed to assess which algorithms would proceed to subsequent phases. All applicable classification algorithms (see Appendix A for a brief description), contained within WEKA 3-9 (developer edition) were performed on one fold of the $32 \mathrm{k} n=1$ dataset, as described in [9]. This allowed a battery of classifiers from across the spectrum of types of be directly compared for the current problem. Parallelizable algorithms are denoted with classifier_name(4), indicating four cores were allocated for execution. This dataset had previously been shown to provide the optimal classification accuracies [9]. The default hyperparameters were used for each algorithm during the coarse search phase. The default parameter values within WEKA have been set to values which have been empirically shown to provide a broad level of performance across different datasets [26]. While parameter tuning is always a key step in a machine learning process, this can introduce an optimistic bias into the experiment, where the classifier is tuned to maximise performance on the test data. Further, the time demand for tuning classifiers can be in the order of weeks, so for phase one, the default parameters were retained. 10-fold crossvalidation was used for internal validation, and 10 iterations of each run were completed so that the results of the nondeterministic classifiers could be averaged. This gave a total of 100 runs across each classifier, with each data instance being used as a training and testing sample 10 times.

The initially intended process was to examine the performance of the classifiers within phase one, and identify a subset to proceed to the next phase based on averaged accuracy. In order to apply a rigorous statistical selection procedure, an ANOVA with paired t-test was conducted on accuracy (correctly classified instances). This compared the means across multiple populations (classifiers), comparing each to check if any of the population means were significantly different from others. This analysis failed to provide any insight, as all distributions were significantly different from all others. 

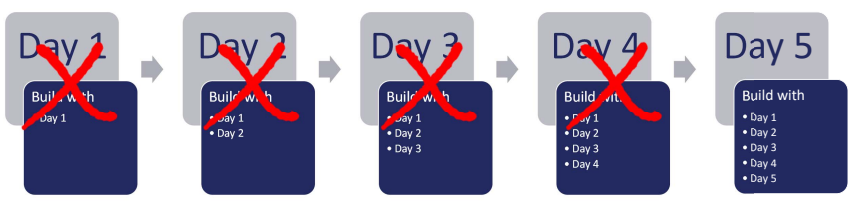

Fig. 2: Non-updatable model updating problem

Only two of the classifiers performed below a standard deemed acceptable; ZeroR had an accuracy asymptotic to random chance (50\%); MultiLayerPerceptron, which took 28 days to complete the training and testing on iteration one alone. Considering phase two involved searching for the optimal parameters using cross-validation, and MultilayerPerceptron contains 8 parameters, it was decided that tuning would take an inappropriate length of time, and so the classifier was removed from the next phase.

2) Phase 2: Hyper-parameter Tuning: Each classifier, selected from phase one for use in phase two, was processed through the CVParameterSelection function in WEKA [27], using a hold-out of $66 \%$ training and $33 \%$ testing. As the CVParameterSelection function performs a parameter search using an internal 10-fold CV, no external CV was implemented. The aim of this phase was to uncover the optimal parameters for each classifier, based on highest percentage correct value. The classifiers were then passed to phase three with their tuned parameters over-riding the defaults.

3) Phase 3: Tuned Classifier Performance: For the third phase, experimental conditions were held constant for each classifier, so that direct comparisons could be made on their performance. Each tuned classifier was implemented using the Experimenter interface of WEKA, running on a remote dedicated server-class machine hosting a 12-core Intel Xeon X5650 2.67GHz CPU and 96GB RAM. A dedicated machine was used so that the performance was as isolated as possible from the effects of other processes on the machine, which may have impacted on performance. The Java Virtual Machine used by WEKA was limited to 8GB of RAM and no more than four cores were explicitly called for the parallelizable algorithms. This approach was taken so that the training and testing processes were performed on what could be considered a standardly specified machine, which will be discussed further in the conclusions of this paper. Classifiers were trained and tested sequentially, using 10 -fold $\mathrm{CV}$ on the same dataset as employed on previous phases.

4) Phase 4: Updating: All models implementing the interface weka.classifiers. Updatableclassifier are capable of incremental learning. Of the classifiers implemented in this phase, HoeffdingTree, IBk, KStar and LWL were updatable. The other classifiers were considered non-incremental and therefore not updatable.

This phase simulated the growth in malware samples by using five new datasets to examine the costs of rebuilding classifiers on escalating datasets. As shown in figure 2, nonupdatable classifiers must discard the pre-learned model if new learning data is to be added. Conversely, updatable classifiers

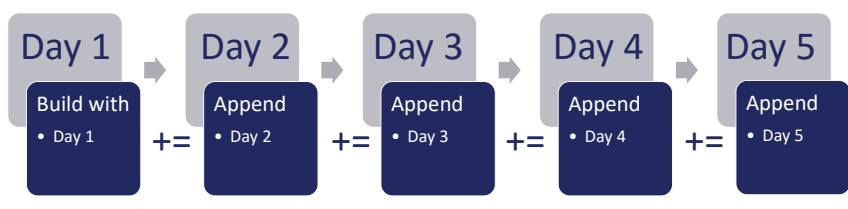

Fig. 3: Updatable model incrementation

can simply add new instances in batches to evolve the existing model, as shown in figure 3.

The dataset from the previous phases was randomly sampled into six stratified folds, i.e. the class ratio was kept exactly the same throughout each fold, five training folds and a separate testing-only fold. A separate test set was required so that direct comparisons could be made between classifier performances, as non-updatable classifiers would be tested on different data if a holdout strategy was employed. The process is stated in Algorithm 1.

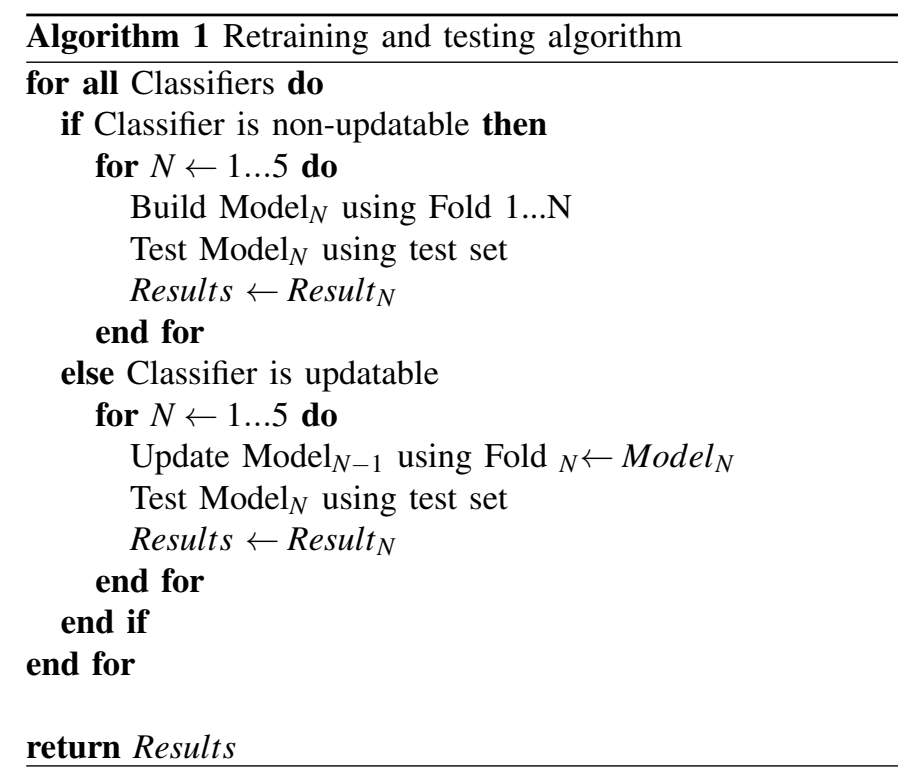

\section{B. Measurements}

There are several methods of measuring process times for applications:

1) User CPU Time: time the thread used in actual CPU cycles, i.e. how long the computer spent doing the calculations requested.

2) Elapsed Time: the difference between system times at the start and end of the requested calculations, including all other processes ( $\mathrm{I} / \mathrm{O}$, disk-read, networking etc) running on the machine (i.e. wall-clock time).

3) System Time: time the OS took responding to the application's requests.

For the current research, User CPU time was used for timing measurements, as it is a direct measurement of the time taken by the actual machine learning process. If a separate CPU-intense process runs at the same 
time, 'elapsed time' will be affected and produce different results each time. As the algorithms were implemented in bespoke Java code, programmed to the WEKA API, the exact measurements could be taken at the specific training/testing times. To measure the training and testing times, the ThreadMXBean interface of the Java API was employed. The ThreadMXBean.get ThreadCPUtime () method was used directly before and after the call to train and the call to test each classifier and the results recorded. The experiment was rerun five times, to average out the timing measurements and mitigate interference which had not been controlled for, such as JVM start-up time.

\section{RESULTS}

\section{A. Phase 1: Classification with default parameters}

1) Classification accuracies: Standard machine learning has multiple measures for defining the accuracy of a predictive model, each with benefits and limitations. For a description of machine learning terms, including accuracy measurements, see Appendix B. Table I illustrates the accuracy measures for phase 1 , ranked by $\%$ correct. There are distinct groupings, with the majority (top 16) achieving $>94.5 \%$ accuracy. The subsequent classifiers range from $89.324 \%$ to $49.953 \%$ i.e. approaching random. The true/false positive/negative ratings $(1=100 \%)$ show the top 16 classifiers can correctly classify either class (benign or malicious) between approximately 95$99 \%$ of the time.

TABLE I: Phase 1 accuracy measures, averaged over 10 runs, ranked by $\%$ correct

\begin{tabular}{lccccc}
\hline Classifier & \% Correct & TPR & FPR & TNR & FNR \\
\hline RandomForest & 99.038 & 0.989 & 0.009 & 0.991 & 0.011 \\
RandomForest(4) & 99.038 & 0.989 & 0.009 & 0.991 & 0.011 \\
RandomCommittee & 98.968 & 0.990 & 0.011 & 0.989 & 0.010 \\
RandomCommittee(4) & 98.968 & 0.990 & 0.011 & 0.989 & 0.010 \\
RandomSubSpace & 98.545 & 0.982 & 0.011 & 0.989 & 0.018 \\
RandomSubSpace(4) & 98.545 & 0.982 & 0.011 & 0.989 & 0.018 \\
PART & 98.366 & 0.983 & 0.016 & 0.984 & 0.017 \\
Bagging & 98.317 & 0.982 & 0.015 & 0.985 & 0.018 \\
Bagging(4) & 98.317 & 0.982 & 0.015 & 0.985 & 0.018 \\
IBk & 98.303 & 0.989 & 0.023 & 0.977 & 0.011 \\
J48 & 98.115 & 0.980 & 0.017 & 0.983 & 0.020 \\
JRip & 97.882 & 0.977 & 0.019 & 0.981 & 0.023 \\
RandomTree & 97.843 & 0.977 & 0.020 & 0.980 & 0.023 \\
REPTree & 97.579 & 0.975 & 0.023 & 0.977 & 0.025 \\
LMT & 97.476 & 0.979 & 0.029 & 0.971 & 0.021 \\
DecisionTable & 94.513 & 0.949 & 0.059 & 0.941 & 0.051 \\
OneR & 89.324 & 0.896 & 0.110 & 0.890 & 0.104 \\
AdaBoostM1 & 88.285 & 0.863 & 0.098 & 0.902 & 0.137 \\
DecisionStump & 81.906 & 0.790 & 0.152 & 0.848 & 0.210 \\
SGD & 76.064 & 0.644 & 0.123 & 0.877 & 0.356 \\
SMO & 72.311 & 0.634 & 0.188 & 0.812 & 0.366 \\
HoeffdingTree & 61.938 & 0.762 & 0.523 & 0.477 & 0.238 \\
ZeroR & 49.953 & 0.500 & 0.500 & 0.500 & 0.500 \\
\hline
\end{tabular}

Table II lists the performance ratios, ranked by precision (true positive class out of predicted positive class). While class imbalance would suggest using the AU-PRC as a preferred indicator of accuracy, this does not take into consideration the negative classifications. As the class imbalance is in the positive class (i.e. more malicious samples), this may distort the context of the measure. Using the AU-ROC, excellent values can be observed for the top 16 classifiers, approaching a maximum score (1). In this context, the MCC score is robust despite the class imbalances and indicates the quality of a binary classifier. With a score of $+1(+100 \%)$ indicating a perfect classifier, the top 15 algorithms achieved scores of $>95 \%$, demonstrating high levels of correlation between the predicted class and the observed class. The bottom three algorithms showed poor performance on this metric, with ZeroR achieving a rating of 0 , i.e. no correlation between prediction and observation.

TABLE II: Phase 1 accuracy ratios, averaged over 10 runs, ranked by precision

\begin{tabular}{lcccccc}
\hline Classifier & Precision & Recall & F & MCC & AUROC & AUPRC \\
\hline RandomForest & 0.991 & 0.989 & 0.990 & 0.981 & 0.999 & 0.999 \\
RandomForest(4) & 0.991 & 0.989 & 0.990 & 0.981 & 0.999 & 0.999 \\
RandomCommittee & 0.989 & 0.990 & 0.990 & 0.979 & 0.998 & 0.998 \\
RandomCommittee(4) & 0.989 & 0.990 & 0.990 & 0.979 & 0.998 & 0.998 \\
RandomSubSpace & 0.989 & 0.982 & 0.985 & 0.971 & 0.998 & 0.999 \\
RandomSubSpace(4) & 0.989 & 0.982 & 0.985 & 0.971 & 0.998 & 0.999 \\
Bagging & 0.985 & 0.982 & 0.983 & 0.966 & 0.998 & 0.998 \\
Bagging(4) & 0.985 & 0.982 & 0.983 & 0.966 & 0.998 & 0.998 \\
PART & 0.984 & 0.983 & 0.984 & 0.967 & 0.989 & 0.985 \\
J48 & 0.983 & 0.980 & 0.981 & 0.962 & 0.985 & 0.979 \\
JRip & 0.981 & 0.977 & 0.979 & 0.958 & 0.984 & 0.978 \\
RandomTree & 0.980 & 0.977 & 0.978 & 0.957 & 0.979 & 0.973 \\
IBk & 0.977 & 0.989 & 0.983 & 0.966 & 0.988 & 0.980 \\
REPTree & 0.977 & 0.975 & 0.976 & 0.952 & 0.989 & 0.985 \\
LMT & 0.971 & 0.979 & 0.975 & 0.950 & 0.984 & 0.976 \\
DecisionTable & 0.942 & 0.949 & 0.945 & 0.891 & 0.983 & 0.981 \\
AdaBoostM1 & 0.899 & 0.863 & 0.880 & 0.767 & 0.954 & 0.948 \\
OneR & 0.892 & 0.896 & 0.894 & 0.787 & 0.893 & 0.851 \\
SGD & 0.840 & 0.644 & 0.729 & 0.536 & 0.761 & 0.719 \\
DecisionStump & 0.839 & 0.790 & 0.814 & 0.639 & 0.819 & 0.768 \\
SMO & 0.772 & 0.634 & 0.696 & 0.454 & 0.723 & 0.672 \\
HoeffdingTree & 0.694 & 0.762 & 0.633 & 0.338 & 0.576 & 0.568 \\
ZeroR & 0.250 & 0.500 & 0.333 & 0.000 & 0.500 & 0.500 \\
\hline
\end{tabular}

The context of the classification ratings, controlled for random chance, is illustrated with table III. Kappa places the achieved agreement in the context of what would be expected by random chance. The top 15 classifiers achieved a Kappa value of $>90 \%$, with the bottom three showing their ratings are not much better than chance alone.

\section{B. Phases 2 \& 3: Hyper-parameter tuning and classification with tuned algorithms}

1) Classification accuracies: Fig. 4 illustrates the effects of tuning the default hyper-parameters on classification accuracy across classifiers i.e. the increase in performance between phase 1 and phase 3 . This is indicated by the line length, i.e. a longer line indicates a larger increase from phase 1 to 3 . Three classifiers showed the greatest increases in accuracy: HoeffdingTree 48.72\%, SMO 31.31\%, and AdaBoostM1 $12.16 \%$, with the other classifiers showing small or negligible performance increases. This can be interpreted in two ways. First, that the default parameters of the classifiers which showed minimal improvement generalized to the current problem well i.e. that tuning was non-essential, whereas the others did not. Second, the classifiers which required tuning to optimize performance were in danger of over-fitting to the current data, which may indicate future performance issues should the training and/or test data change substantially. 


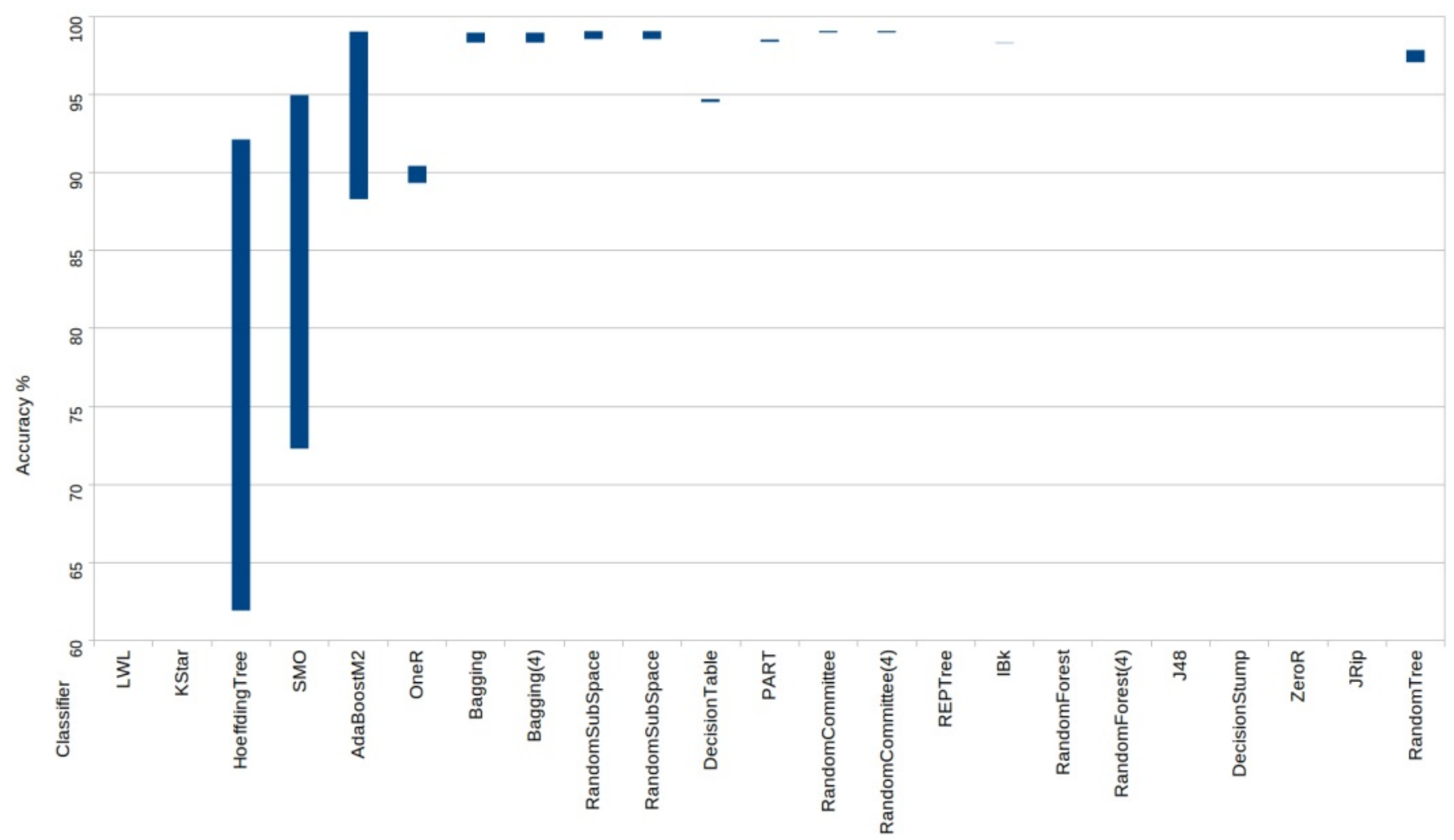

Fig. 4: Effects of hyper-parameter tuning on classification accuracy (Double column width)

TABLE III: Phase 1 Kappa values per classifier

\begin{tabular}{lr}
\hline Classifier & Kappa \\
\hline RandomForest & 0.9808 \\
RandomForest(4) & 0.9808 \\
RandomCommittee & 0.9794 \\
RandomCommittee(4) & 0.9794 \\
RandomSubSpace & 0.9709 \\
RandomSubSpace(4) & 0.9709 \\
PART & 0.9673 \\
Bagging & 0.9663 \\
Bagging(4) & 0.9663 \\
IBk & 0.9661 \\
J48 & 0.9623 \\
JRip & 0.9576 \\
RandomTree & 0.9569 \\
REPTree & 0.9516 \\
LMT & 0.9495 \\
DecisionTable & 0.8903 \\
OneR & 0.7865 \\
AdaBoostM1 & 0.7657 \\
DecisionStump & 0.6381 \\
SGD & 0.5213 \\
SMO & 0.4462 \\
HoeffdingTree & 0.2388 \\
ZeroR & 0 \\
\hline
\end{tabular}

The performance of the classifiers averaged over 10 runs in phase 3 is listed in Table IV, in terms of correctly classified instances. Overall, the classifiers showed excellent classification accuracy, with 21 of the 23 classifiers achieving an accuracy of $>90 \%$, and $8>99 \%$. While Table IV indicates testing was on 1065 instances, this is the averaged figure over each of 10 runs.

Further accuracy ratios for this phase are shown in Table V. The top 10 classifiers present excellent metrics, with low FP $(<1 \%)$, F-score $(\geq 0.990)$, AU-ROC and AU-PRC (0.999 with the exception of AdaBoostM1 (0.994)). These are indicative of high-performing classifiers, capable of discriminating binary classes with high levels of accuracy.

2) Training and testing times: The UserCPU times for training and testing, averaged over the 10 runs are listed in Tables VI and VII. Training times appear lowest for IBk, KStar and LWL, however these classifiers are considered 'lazy' learners with only feature loading occurring during the traditional training phase i.e. no training actually occurs. Parallelized classifiers show very fast learning times (0.0030.01 seconds), as the training phase is well-suited to task parallelization, particularly when compared with non-parallelized equivalents. Testing times show a much more compact distribution, with all classifiers except KStar (4241.640) completing in under 9 seconds. The mean testing time was 194.07 seconds, though excluding KStar greatly reduced this to 1.33 seconds. Parallelization had minimal effect on the testing phase, due to the iterative process for testing on an instance-by-instance 
TABLE V: Accuracy ratios averaged over 10 runs for Phases $2 \& 3$

\begin{tabular}{|c|c|c|c|c|c|c|c|c|c|c|c|}
\hline Classifier & Kappa & TPR & FPR & TNR & FNR & Precision & Recall & F & $\mathrm{MCC}$ & AUROC & AUPRC \\
\hline RandomCommittee & 0.981 & 0.989 & 0.008 & 0.992 & 0.011 & 0.992 & 0.989 & 0.991 & 0.981 & 0.999 & 0.999 \\
\hline RandomCommittee(4) & 0.981 & 0.989 & 0.008 & 0.992 & 0.011 & 0.992 & 0.989 & 0.991 & 0.981 & 0.999 & 0.999 \\
\hline RandomSubSpace & 0.981 & 0.989 & 0.008 & 0.992 & 0.011 & 0.992 & 0.989 & 0.990 & 0.981 & 0.999 & 0.999 \\
\hline RandomSubSpace (4) & 0.981 & 0.989 & 0.008 & 0.992 & 0.011 & 0.992 & 0.989 & 0.990 & 0.981 & 0.999 & 0.999 \\
\hline RandomForest & 0.981 & 0.989 & 0.008 & 0.992 & 0.011 & 0.992 & 0.989 & 0.990 & 0.981 & 0.999 & 0.999 \\
\hline RandomForest(4) & 0.981 & 0.989 & 0.008 & 0.992 & 0.011 & 0.992 & 0.989 & 0.990 & 0.981 & 0.999 & 0.999 \\
\hline AdaBoostM1 & 0.980 & 0.989 & 0.009 & 0.991 & 0.011 & 0.991 & 0.989 & 0.990 & 0.981 & 0.996 & 0.994 \\
\hline AdaBoostM1(4) & 0.980 & 0.989 & 0.009 & 0.991 & 0.011 & 0.991 & 0.989 & 0.990 & 0.981 & 0.996 & 0.994 \\
\hline Bagging & 0.979 & 0.987 & 0.008 & 0.992 & 0.013 & 0.992 & 0.987 & 0.990 & 0.979 & 0.999 & 0.999 \\
\hline Bagging(4) & 0.979 & 0.987 & 0.008 & 0.992 & 0.013 & 0.992 & 0.987 & 0.990 & 0.979 & 0.999 & 0.999 \\
\hline PART & 0.970 & 0.984 & 0.014 & 0.986 & 0.016 & 0.986 & 0.984 & 0.985 & 0.970 & 0.989 & 0.986 \\
\hline IBk & 0.967 & 0.990 & 0.023 & 0.977 & 0.010 & 0.977 & 0.990 & 0.983 & 0.967 & 0.988 & 0.980 \\
\hline LWL & 0.967 & 0.990 & 0.023 & 0.977 & 0.010 & 0.977 & 0.990 & 0.983 & 0.967 & 0.985 & 0.976 \\
\hline $\mathrm{J} 48$ & 0.962 & 0.981 & 0.018 & 0.982 & 0.019 & 0.982 & 0.981 & 0.981 & 0.962 & 0.981 & 0.974 \\
\hline KStar & 0.962 & 0.979 & 0.017 & 0.983 & 0.021 & 0.983 & 0.979 & 0.981 & 0.962 & 0.993 & 0.993 \\
\hline JRip & 0.957 & 0.977 & 0.020 & 0.980 & 0.023 & 0.980 & 0.977 & 0.978 & 0.957 & 0.985 & 0.980 \\
\hline REPTree & 0.953 & 0.974 & 0.022 & 0.978 & 0.026 & 0.978 & 0.974 & 0.976 & 0.953 & 0.990 & 0.987 \\
\hline RandomTree & 0.941 & 0.968 & 0.027 & 0.973 & 0.032 & 0.973 & 0.968 & 0.971 & 0.941 & 0.986 & 0.982 \\
\hline DecisionTable & 0.894 & 0.951 & 0.057 & 0.943 & 0.049 & 0.944 & 0.951 & 0.947 & 0.894 & 0.984 & 0.983 \\
\hline HoeffdingTree & 0.842 & 0.929 & 0.087 & 0.913 & 0.071 & 0.915 & 0.929 & 0.922 & 0.844 & 0.922 & 0.902 \\
\hline OneR & 0.808 & 0.907 & 0.098 & 0.902 & 0.093 & 0.903 & 0.907 & 0.904 & 0.809 & 0.904 & 0.865 \\
\hline DecisionStump & 0.638 & 0.790 & 0.152 & 0.848 & 0.210 & 0.839 & 0.790 & 0.814 & 0.639 & 0.819 & 0.768 \\
\hline ZeroR & 0.000 & 0.500 & 0.500 & 0.500 & 0.500 & 0.250 & 0.500 & 0.333 & 0.000 & 0.500 & 0.500 \\
\hline
\end{tabular}

TABLE IV: Phase 2\&3: Performance of tuned algorithms averaged over 10 runs

\begin{tabular}{llllll}
\hline Classifier & $\begin{array}{l}\text { Number of } \\
\text { training instances }\end{array}$ & $\begin{array}{l}\text { Number of } \\
\text { testing instances }\end{array}$ & $\begin{array}{l}\text { Number } \\
\text { correct }\end{array}$ & $\begin{array}{l}\text { Number } \\
\text { incorrect }\end{array}$ & $\begin{array}{l}\% \\
\text { correct }\end{array}$ \\
\hline RandomCommittee & 9585 & 1065 & 1055 & 10 & 99.061 \\
RandomCommittee(4) & 9585 & 1065 & 1055 & 10 & 99.061 \\
RandomSubSpace & 9585 & 1065 & 1054.9 & 10.1 & 99.052 \\
RandomSubSpace & 9585 & 1065 & 1054.9 & 10.1 & 99.052 \\
RandomForest & 9585 & 1065 & 1054.9 & 10.1 & 99.052 \\
RandomForest(4) & 9585 & 1065 & 1054.9 & 10.1 & 99.052 \\
AdaBoostM1 & 9585 & 1065 & 1054.6 & 10.4 & 99.023 \\
AdaBoostM1 & 9585 & 1065 & 1054.6 & 10.4 & 99.023 \\
Bagging & 9585 & 1065 & 1053.9 & 11.1 & 98.958 \\
Bagging(4) & 9585 & 1065 & 1053.9 & 11.1 & 98.958 \\
PART & 9585 & 1065 & 1049.1 & 15.9 & 98.507 \\
IBk & 9585 & 1065 & 1047.2 & 17.8 & 98.329 \\
LWL & 9585 & 1065 & 1047.2 & 17.8 & 98.329 \\
J48 & 9585 & 1065 & 1045 & 20 & 98.122 \\
KStar & 9585 & 1065 & 1044.7 & 20.3 & 98.094 \\
JRip & 9585 & 1065 & 1041.9 & 23.1 & 97.831 \\
REPTree & 9585 & 1065 & 1039.8 & 25.2 & 97.634 \\
RandomTree & 9585 & 1065 & 1033.7 & 31.3 & 97.061 \\
DecisionTable & 9585 & 1065 & 1008.5 & 56.5 & 94.695 \\
HoeffdingTree & 9585 & 1065 & 981 & 84 & 92.113 \\
OneR & 9585 & 1065 & 963 & 102 & 90.423 \\
DecisionStump & 9585 & 1065 & 872.3 & 192.7 & 81.906 \\
ZeroR & 9585 & 1065 & 532 & 533 & 49.953 \\
\hline
\end{tabular}

basis.

3) Serialized model sizes: As WEKA is developed in Java, the ability to implement the java.io.Serializable interface means trained models can be serialized in persistent storage, then called to classify future instances. Fig.5 depicts the size on disk of the serialized trained models for each algorithm in phase 3 . RandomSubspace produced by far the largest model $\sim 103 \mathrm{MB}$, with RandomCommittee (30.8MB), Bagging (26.7MB), and AdaBoostM1 (17.1MB) also creating larger models than the other algorithms. Each of these classifiers is an ensemble-based learner, using multiple sub-classifiers to create overall models, and therefore larger serialized sizes. However, RandomForest is also an ensemble learner of trees, but placed around the mid-rank level of the classifiers.

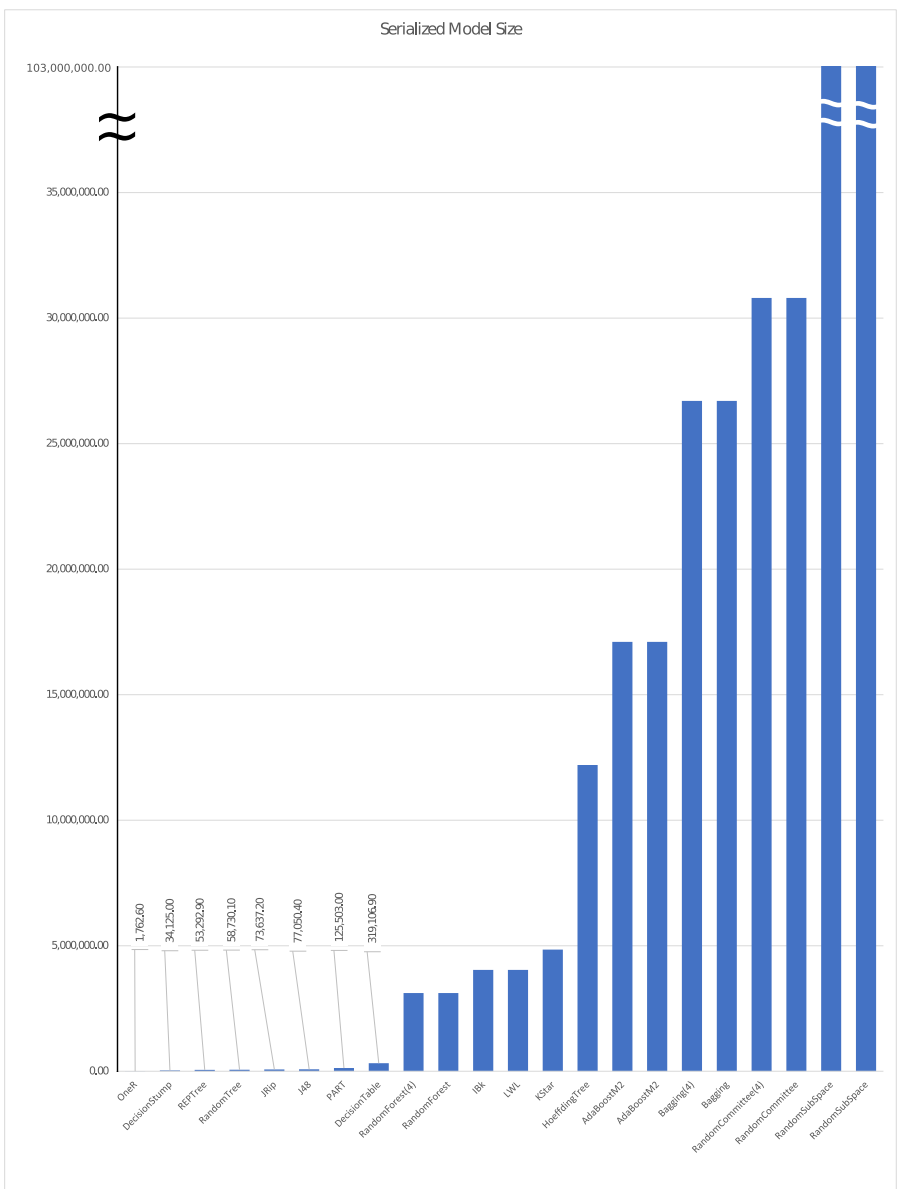

Fig. 5: Serialized model sizes (bytes)(half page width) 
TABLE VI: Phase 2\&3: UserCPU Training time (s)

*Lazy learners do not have traditional training phases

\begin{tabular}{lr}
\hline Classifier & Time \\
\hline IBk* & 0.001 \\
KStar* & 0.001 \\
LWL* & 0.002 \\
Bagging(4) & 0.003 \\
RandomCommittee(4) & 0.005 \\
RandomForest(4) & 0.009 \\
RandomSubSpace(4) & 0.010 \\
RandomTree & 0.342 \\
OneR & 1.191 \\
DecisionStump & 1.697 \\
HoeffdingTree & 5.845 \\
REPTree & 7.698 \\
J48 & 17.706 \\
AdaBoostM1(4) & 18.914 \\
PART & 32.609 \\
RandomForest & 38.014 \\
DecisionTable & 102.845 \\
AdaBoostM1 & 187.411 \\
JRip & 221.719 \\
Bagging & 346.898 \\
RandomCommittee & 376.129 \\
RandomSubSpace & 1090.683 \\
\hline
\end{tabular}

TABLE VII: Phase 2\&3: UserCPU Testing time (s)

\begin{tabular}{lr}
\hline Classifier & Time \\
\hline OneR & 0.001 \\
DecisionStump & 0.001 \\
REPTree & 0.001 \\
J48 & 0.001 \\
JRip & 0.002 \\
PART & 0.003 \\
RandomTree & 0.004 \\
DecisionTable & 0.036 \\
RandomForest(4) & 0.114 \\
RandomForest & 0.115 \\
HoeffdingTree & 0.185 \\
AdaBoostM1(4) & 1.046 \\
AdaBoostM1(4) & 1.087 \\
Bagging & 1.221 \\
Bagging(4) & 1.224 \\
IBk & 1.245 \\
RandomCommittee(4) & 1.258 \\
RandomCommittee & 1.269 \\
RandomSubSpace (4) & 4.883 \\
RandomSubSpace & 5.685 \\
LWL & 8.625 \\
KStar & 4241.640 \\
\hline
\end{tabular}

TABLE VIII: Phase 4: Accuracy (\% correct) across training subsets, ranked by $n=43530$

\begin{tabular}{lllllll}
\hline Rank & Classifier & Accuracies & & & & \\
\hline & & $n=8706$ & $n=17412$ & $n=26118$ & $n=34824$ & $n=43530$ \\
1 & AdaBoostM1 & 99.2649 & 99.9196 & 99.9081 & 99.9081 & 99.9081 \\
- & RandomForest & 99.2878 & 99.9081 & 99.9081 & 99.8966 & 99.9081 \\
- & RandomForest(4) & 99.2878 & 99.9081 & 99.9081 & 99.8966 & 99.9081 \\
- & RandomSubSpace(4) & 99.2993 & 99.9081 & 99.9081 & 99.9081 & 99.9081 \\
- & AdaBoostM1(4) & 99.2649 & 99.9196 & 99.9081 & 99.9081 & 99.9081 \\
- & RandomSubSpace & 99.2993 & 99.9081 & 99.9081 & 99.9081 & 99.9081 \\
- & RandomCommittee & 99.3453 & 99.9081 & 99.9081 & 99.9081 & 99.9081 \\
- & RandomCommittee(4) & 99.3453 & 99.9081 & 99.9081 & 99.9081 & 99.9081 \\
2 & Bagging(4) & 99.2304 & 99.8277 & 99.8737 & 99.8622 & 99.8851 \\
- & Bagging & 99.2304 & 99.8277 & 99.8737 & 99.8622 & 99.8851 \\
3 & J48 & 98.8399 & 99.7703 & 99.7818 & 99.8507 & 99.8047 \\
4 & PART & 98.8743 & 99.7588 & 99.7588 & 99.8277 & 99.7818 \\
5 & JRip & 98.8514 & 99.5750 & 99.4831 & 99.5405 & 99.6439 \\
6 & REPTree & 98.3230 & 99.4257 & 99.5635 & 99.5865 & 99.5635 \\
7 & RandomTree & 98.2541 & 99.0237 & 98.9433 & 99.3568 & 99.3797 \\
8 & IBk & 98.8857 & 99.2074 & 99.3222 & 99.2993 & 99.3567 \\
- & LWL & 98.8857 & 99.2074 & 99.3222 & 99.2993 & 99.3567 \\
9 & HoeffdingTree & 97.2659 & 97.1855 & 97.6221 & 97.6565 & 98.0701 \\
10 & DecisionTable & 95.9453 & 96.7838 & 96.8527 & 96.0372 & 96.6000 \\
11 & OneR & 93.8548 & 95.4629 & 95.8419 & 96.0487 & 96.2095 \\
12 & DecisionStump & 92.7981 & 92.7981 & 92.7981 & 92.7981 & 92.7981 \\
13 & ZeroR & 89.8116 & 89.8116 & 89.8116 & 89.8116 & 89.8116 \\
- & KStar & 98.7250 & 99.8507 & 99.8507 & - & - \\
\hline
\end{tabular}

\section{Phase 4: Retraining costs}

1) Accuracies: The accuracy of each classifier was taken at each escalation point i.e. after training on each new dataset. Testing was held constant with a separate testing set, so that the results of the incrementable and non-incrementable classifiers could be directly compared (i.e. they were being tested against the same data). Table VIII lists the accuracies, ordered by the performance following the peak training dataset $(43,530$ instances). KStar was discontinued after dataset 3 due to the unacceptably long training times. The top 17 classifiers had accuracies $>99.35 \%$, with the top 4 ( 8 including parallelized) tied for first rank (99.9081\%). These top classifiers also show consistency across the second to fifth datasets, following an improvement between datasets one and two. There are two slightly anomalous results: AdaBoostM1 at dataset two and RandomForest at dataset three, though these represent +1 and -1 correctly-classified instances respectively.

2) Training and testing times: Fig.6 depicts the training and testing times across each of the five datasets. Although the testing set was held constant, the testing times differ due to the algorithmic complexities of each individual classifier. RandomForest $(4)$ had the lowest training time by dataset five, with other parallelized ensemble learners (RandomCommittee(4) \& Bagging(4)) only $1 \mathrm{~ms}$ slower. Training times typically escalated sharply with the escalating datasets in the majority of classifiers. RandomSubspace(4) was the only classifier which declined overall from the first increment, and was at $50 \%$ by the final increment.

The updatable classifiers showed mixed performance across the escalating data. Hoeffding Tree training time increased, though was relatively stable from datasets two to five. IBk was approximately four times slower at dataset two compared to dataset one, which was maintained across the other datasets. KStar could not complete the five increments in a reasonable time. LWL was 14 times slower at the second increment compared to the first, but this remained stable across each 


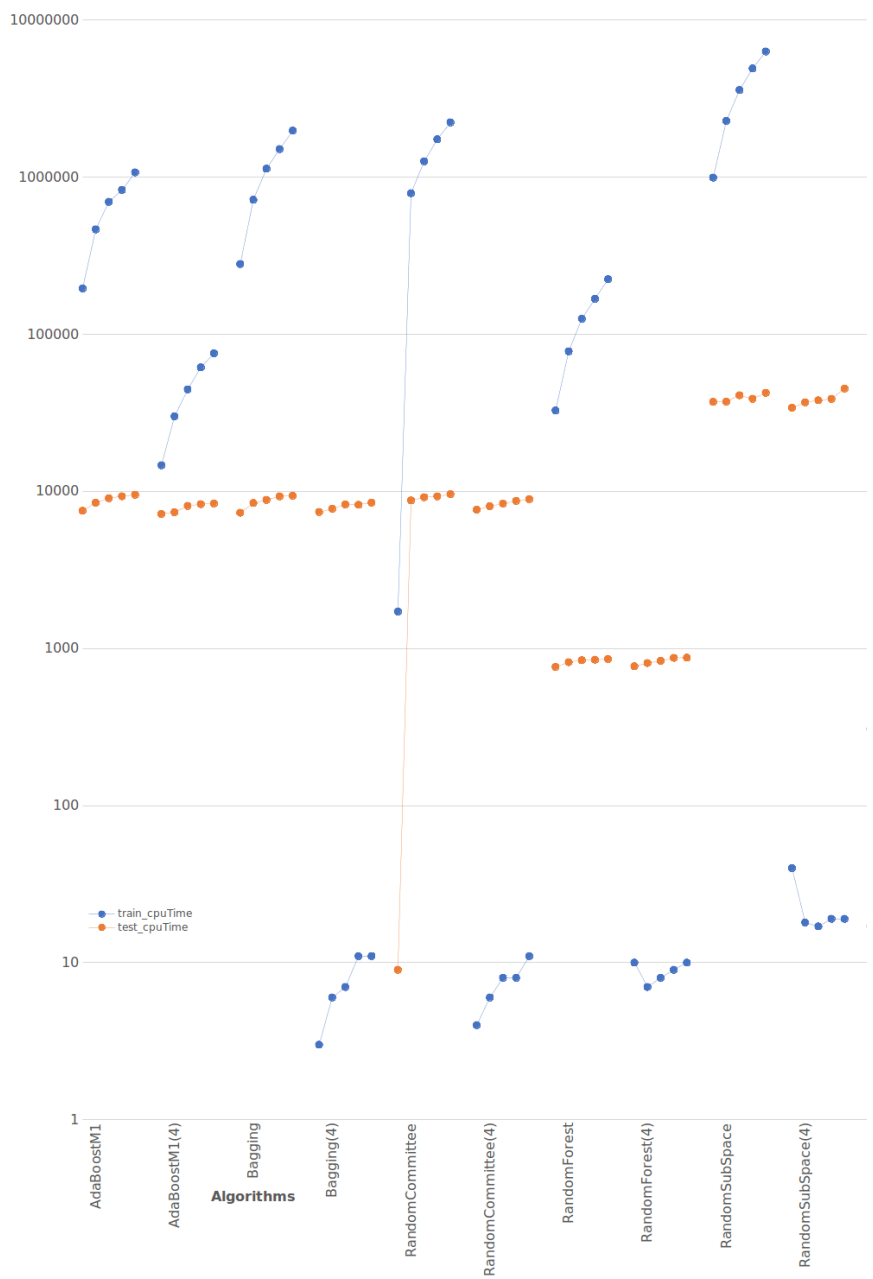

Fig. 7: Phase 4: Effects of parallelization on training and testing times

other increment.

Testing times showed less time increase overall, as the testing set remained at a fixed size. LWL and IBk were notable exceptions ( 6.8 and 5.5 times slower between the first and final increments), due to being lazy learners. HoeffdingTree testing times dropped by a third over the third and fourth dataset, and the fifth was approximately $20 \%$ lower than the initial testing. DecisionStump and REPTree had the two fastest testing times, particularly in reference to their training times, which will be discussed further below.

A key point to note is that parallelized algorithms had only slightly lower testing times, but a marked reduction on the training times of the models. Fig. 7 shows the training and testing times of each of the parallelizable algorithms beside their un-parallelized instances.

3) Consideration of training and testing times with accuracy: The examination of accuracy against both training and testing times is presented in fig.8. Visually, there is a clear separation between the top performing classifiers across all three measures. The remaining classifiers may achieve high performance on one or two measures, but not in a third. For example, LWL has fast training time (as a lazy learner), high

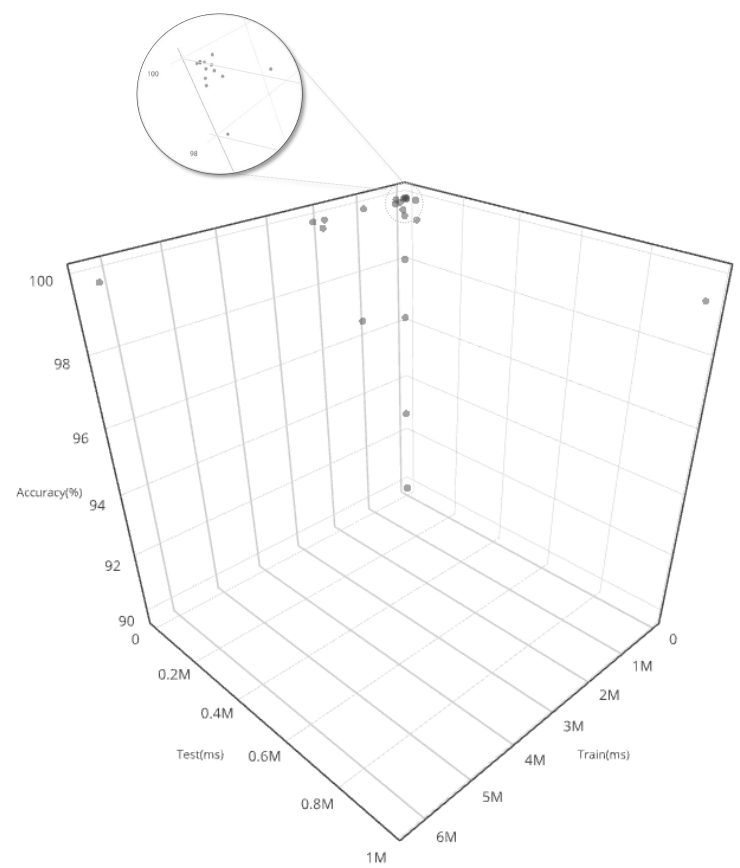

Fig. 8: Training and testing times (ms) against accuracy(correctly classified instances\%)

accuracy, but the slowest testing time out of all classifiers. Interpretation of these measures is based on context and desired results within that context, as will be discussed further in the summary of this chapter.

4) Overall Rankings: As noted in the previous section, the analysis of classifiers across three measures must be interpreted into the context in which they are to be implemented. However, to assess the classifiers across all three measures (training time, testing time, and accuracy), a composite rankbased indicator was created. Each classifier was ranked across each measure and the sum of the ranks formed the overall score i.e. the lowest overall score was the highest ranked on average across all measures.

RandomForest(4) was clearly ranked ahead of all other classifiers across the three measures. It was the joint most accurate and fastest to train. However, it was below the midway ranking point for testing time i.e. half of all classifiers were faster. This indicates the bottleneck of testing without implementation of parallelization In second position, RandomCommittee(4) was joint most accurate, joint second fastest to train and 15 th fastest to test. However the test time was in excess of 10 times slower than RandomForest(4). The other classifiers were reasonably tightly packed in the remaining positions, with only 21 ranking points separating 20 classifiers, with several ties.

\section{Discussion}

\section{A. Phase 1: Classification using default parameters}

In phase one, the performances of 23 classifiers were analysed using ten-times ten-fold cross-validation, initially with default parameters. The top 16 classifiers showed highly accurate discrimination between malware and benignware, again 


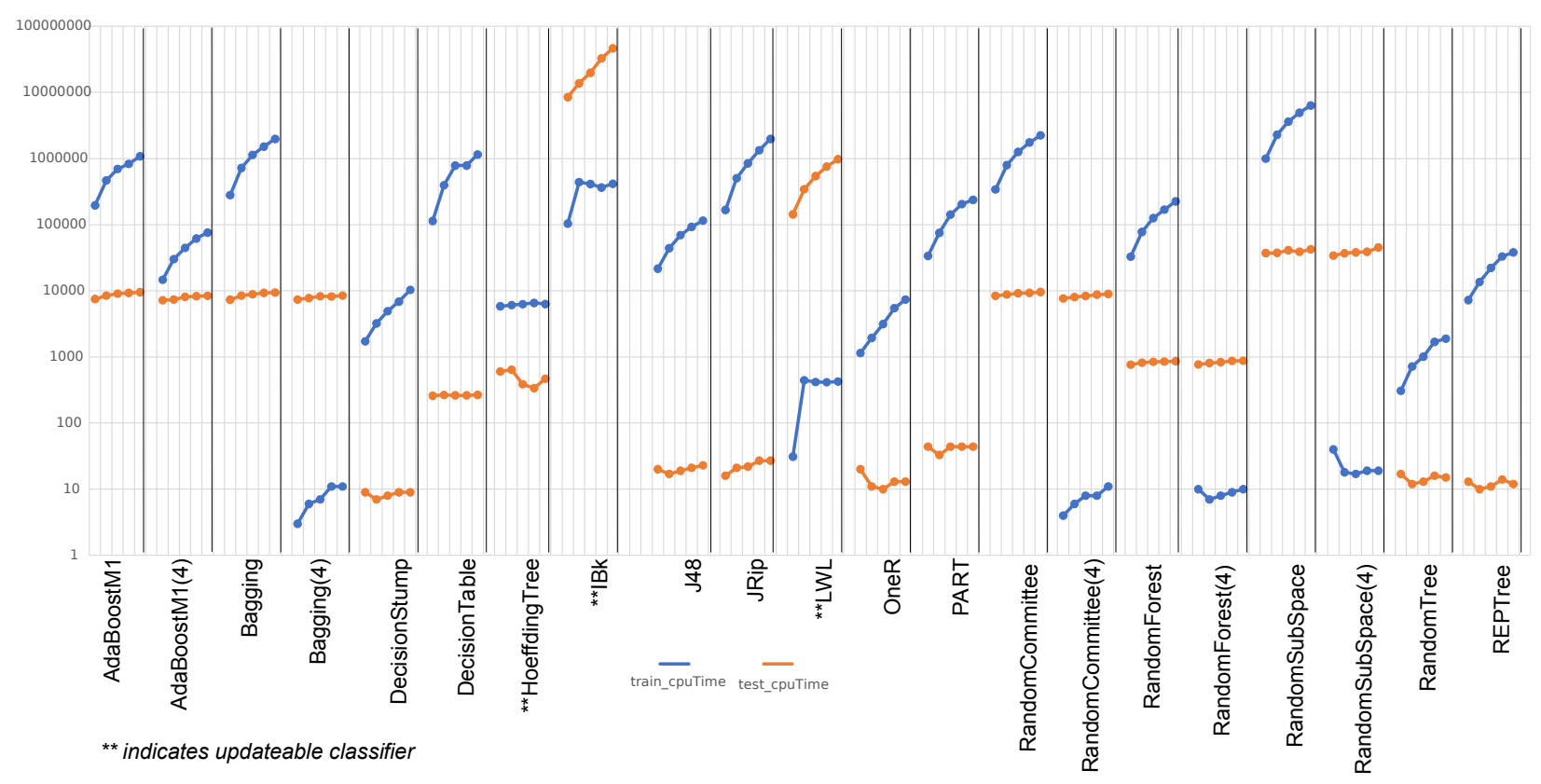

Fig. 6: Training and testing times across 5 escalating datasets per classifier (ms)- log scale

TABLE IX: Phase 4: Ranked Training, Testing and Accuracy

\begin{tabular}{lrrrrrrrr}
\hline Classifier & $\begin{array}{l}\text { UserCPU } \\
\text { Time }\end{array}$ & $\begin{array}{l}\text { Rank } \\
\text { Training }\end{array}$ & $\begin{array}{l}\text { UserCPU } \\
\text { Time } \\
\text { Testing }\end{array}$ & Rank & Accuracy & Rank & $\begin{array}{l}\text { Overall } \\
\text { Score }\end{array}$ & $\begin{array}{l}\text { Overall } \\
\text { Rank }\end{array}$ \\
\hline RandomForest(4) & 10 & $\mathbf{1}$ & 874 & $\mathbf{1 2}$ & 99.9081 & $\mathbf{1}$ & 14 & $\mathbf{1}$ \\
RandomCommittee(4) & 11 & $\mathbf{2}$ & 8932 & $\mathbf{1 5}$ & 99.9081 & $\mathbf{1}$ & 18 & $\mathbf{2}$ \\
Bagging(4) & 11 & $\mathbf{2}$ & 8474 & $\mathbf{1 4}$ & 99.8851 & $\mathbf{9}$ & 25 & $\mathbf{3}$ \\
RandomSubSpace(4) & 19 & $\mathbf{5}$ & 45133 & $\mathbf{2 0}$ & 99.9081 & $\mathbf{1}$ & 26 & $\mathbf{4}$ \\
RandomForest & 224147 & $\mathbf{1 5}$ & 858 & $\mathbf{1 1}$ & 99.9081 & $\mathbf{1}$ & 27 & $\mathbf{5}$ \\
AdaBoostM1(4) & 75588 & $\mathbf{1 3}$ & 8380 & $\mathbf{1 3}$ & 99.9081 & $\mathbf{1}$ & 27 & $\mathbf{5}$ \\
RandomTree & 1893 & $\mathbf{8}$ & 15 & $\mathbf{5}$ & 99.3797 & $\mathbf{1 5}$ & 28 & $\mathbf{7}$ \\
REPTree & 38127 & $\mathbf{1 2}$ & 12 & $\mathbf{2}$ & 99.5635 & $\mathbf{1 4}$ & 28 & $\mathbf{7}$ \\
ZeroR & 13 & $\mathbf{4}$ & 14 & $\mathbf{4}$ & 89.8116 & $\mathbf{2 2}$ & 30 & $\mathbf{9}$ \\
J48 & 114754 & $\mathbf{1 4}$ & 23 & $\mathbf{6}$ & 99.8047 & $\mathbf{1 1}$ & 31 & $\mathbf{1 0}$ \\
DecisionStump & 10341 & $\mathbf{1 1}$ & 9 & $\mathbf{1}$ & 92.7981 & $\mathbf{2 1}$ & 33 & $\mathbf{1 1}$ \\
OneR & 7351 & $\mathbf{1 0}$ & 13 & $\mathbf{3}$ & 96.2095 & $\mathbf{2 0}$ & 33 & $\mathbf{1 1}$ \\
AdaBoostM1 & 1073905 & $\mathbf{1 7}$ & 9515 & $\mathbf{1 7}$ & 99.9081 & $\mathbf{1}$ & 35 & $\mathbf{1 3}$ \\
PART & 237611 & $\mathbf{1 6}$ & 44 & $\mathbf{8}$ & 99.7818 & $\mathbf{1 2}$ & 36 & $\mathbf{1 4}$ \\
HoeffdingTree & 6305 & $\mathbf{9}$ & 470 & $\mathbf{1 0}$ & 98.0701 & $\mathbf{1 8}$ & 37 & $\mathbf{1 5}$ \\
JRip & 1977517 & $\mathbf{1 9}$ & 27 & $\mathbf{7}$ & 99.6439 & $\mathbf{1 3}$ & 39 & $\mathbf{1 6}$ \\
RandomCommittee & 2234350 & $\mathbf{2 1}$ & 9603 & $\mathbf{1 8}$ & 99.9081 & $\mathbf{1}$ & 40 & $\mathbf{1 7}$ \\
RandomSubSpace & 6321037 & $\mathbf{2 2}$ & 42373 & $\mathbf{1 9}$ & 99.9081 & $\mathbf{1}$ & 42 & $\mathbf{1 8}$ \\
IBk & 420 & $\mathbf{6}$ & 48832 & $\mathbf{2 1}$ & 99.3567 & $\mathbf{1 6}$ & 43 & $\mathbf{1 9}$ \\
Bagging & 1979406 & $\mathbf{2 0}$ & 9389 & $\mathbf{1 6}$ & 99.8851 & $\mathbf{9}$ & 45 & $\mathbf{2 0}$ \\
LWL & 424 & $\mathbf{7}$ & 975107 & $\mathbf{2 2}$ & 99.3567 & $\mathbf{1 6}$ & 45 & $\mathbf{2 0}$ \\
DecisionTable & 1149883 & $\mathbf{1 8}$ & 267 & $\mathbf{9}$ & 96.6001 & $\mathbf{1 9}$ & 46 & $\mathbf{2 2}$ \\
\hline
\end{tabular}

supporting the idea that opcode analysis is a viable detection method. As default parameters were used in this phase, and the top performing classifier scored $99.038 \%$ accuracy, this would suggest that not only are the default parameters broadly acceptable, but a computationally-expensive tuning phase is not automatically needed for this classifier. This minimizes the risk of over-fitting the tuned classifier to the data at hand, enhancing its extrapolability to future data. This is the first cross-classifier comparison of dynamic opcode runtraces on a dataset of this scale.

\section{B. Phases 2 \& 3: Hyperparameter tuning and classification using optimal parameters}

Phase two featured tuning the hyper-parameters of the algorithms to increase accuracy. Once the classifiers were tuned, phase 3 then examined the performances. For the majority of classifiers, no significant increase was found between defaultparameter and tuned-parameter classifiers. However, three classifiers improved drastically. HoeffdingTree's accuracy improved by nearly half, which may indicate over-fitting to the present data. This is exacerbated in phase four, which will be discussed further below. The tuning phase is computationally expensive, and can take a substantial amount of time. Any implementation of this in a real life setting would falter at this period, with weeks or even months of data accumulating in the tuning period, rendering the model immediately obsolete prior to being built. An optimal scenario would involved an algorithm which does not require tuning, or can do so in a short period of time. The classifiers showed excellent levels of accuracy in this phase, with the top eight scoring in excess of 99\% accuracy, with low false positive rates. All eight are ensemble learners, with RandomForest as a base classifier. This indicates the power of tree-based algorithms, coupled with the accuracy-boosting which can be gained from ensemble techniques. However, ensembling strategies can suffer performance penalties, particularly when parallelization is not utilized With regards training time, unparallelized versions of the top 8 classifiers occupied four of the bottom five ranks. As parallelization is not utilized in testing, the parallelized and unparallelized versions of the eight ensemble learners were in the bottom 11, i.e. the slowest half. This demonstrates 
not only the benefits of parallelization in ensemble learning, but the trade-offs between accuracy and time. The ensemble classifiers, by nature, also had the largest model sizes on disk, although from an implementation perspective this may not cause much of an issue, as all but RandomSubSpace took up less than $31 \mathrm{MB}$.

\section{Phase 4: The costs of retraining vs updating models}

The costs of simulated retraining were examined in phase 4 . As malware data grows on an exponential basis, any machine learning model designed for an actual implementation must take this into account, yet this is not seen in the literature. When datasets double every few days, any implemented model must be capable of retraining within satisfactory times, and while maintaining or growing accuracy. The ability of certain classifiers to be incrementally updated was compared with the total-rebuild time of non-updatable classifiers. Initial thoughts may suggest that incrementable classifiers will be faster over escalating data, however this was not found to be the case. KStar could not be continued after the third dataset, due to unacceptable testing times. HoeffdingTree was 9th most accurate, while IBK and LWL were joint eighth. If the training time was exceptional, this may be acceptable, as they were still highly accurate. However, the updatables came 9th, 7th, and 6th for training, with HoeffdingTree taking longer than the eight algorithms above it combined. In testing, the updatable classifiers occupied ranks 10,22, and 21, indicating that they were generally slower than all or most other classifiers. When the change in accuracies is examined between phases 1 and 3- it may be the case that HoeffdingTree had over-fitted to the current data. This would become an issue with escalating datasets, as the model performance would suffer as the data changes. Hoeffding Tree-based classifiers are correlated to, at worst, the number of attributes. In the present work, the classifier may have benefited from feature reduction methods. Further, VFDTs offer strong guarantees of accuracy performance asymptotic to batch classifiers, given enough samples. It may be entirely possible that our implementation simply did not provide Hoeffding Tree with enough samples to generate a robust tree and, given more samples, the accuracy could be improved.

Further, it is worth noting that HoeffdingTree caused some implementation issues during the experiment. As an updatable classifier, Hoeffding Trees only work when incoming data is presented in the same ratio as they have been trained on i.e. the class balance must remain constant. Also, as discovered during the testing phase, the instance order must be randomized when presented to the classifier for training. During the testing experiments, HoeffdingTree had accuracies approaching random chance, which was anomalous to other results. The issue stemmed from the straight-loading of the datasets, rather than cross-validation fold making, despite an initial stratified folds approach. As the datasets presented the classes in order i.e. all benign followed by all malicious, HoeffdingTree was greatly affected. Upon investigation, [10] explains that the first instances the learner encounters are used to choose the attribute on which to split at the root.
Subsequent instances pass through the tree until they reach a leaf, at which point they are employed to choose the attribute to split on. Randomizing the order of the instances resulted in the accuracies presented. These two factors have serious implications for attempts at implementing the algorithm in an applied setting. While malware will grow exponentially, benignware is much harder to access, and so artificial means may have to be employed to ensure the data is in the same class-balance. Further, the instances presented must be in a randomized order, which would cause difficulties in a data streaming-based approach.

\section{Conclusions}

This paper presents the analyses of 23 classifiers in the context of malware detection using runtime opcodes. While machine learning approaches to dynamic opcode analysis have been covered in the literature, no studies, to the best of the author's knowledge, have been conducted into the costs of training and testing algorithms in this context. Further, while each paper in the literature highlights the exponential growth in malware, the datasets for each remain fixed. No investigation has been made into the effects of escalating data on these models, both in terms of accuracy and training or testing time.

It is clear that parallelized ensemble classifiers offer the best performance in terms of training and accuracy, with 5 such algorithms taking the top five ranks, with a non-parallelized ensemble technique taking joint 5th (RandomForest). While testing times are typically slower, it may be beneficial to investigate parallelized testing in future work. However, if the summed ranking approach is applied to only testing and accuracy, RandomForest and RandomForest(4) occupy the first and second ranks. From an implementation perspective, context is key, but the results of the above analyses are clear. If training speed and accuracy are most important in the implementation, RandomForest(4) is the clear top performer. If testing time and accuracy are key, unparallelized RandomForest is the ideal classifier, though the parallelized version is only $16 \mathrm{~ms}$ slower. When all three measures are taken into account, parallelized RandomForest presents as the clear choice. However, with further increases in data in the order of months or years, this may not continue to hold true. Further work is required to investigate this, and mitigate any disadvantages for use in an applied setting.

To highlight why CPU time in terms of training and testing is important, it is worth putting this question within an applied context i.e. an implementation of the algorithms examined. If these classifiers were implemented within a third-party cloud service, the tangible cost difference could be significant. A c5.xlarge 4 core 8GB Linux Amazon Web Services instance currently costs $\$ 0.192$ per instance hour ${ }^{1}$. Using this as a reference, the fastest classifier RandomForest(4) would cost $\$ 0.000001$ USD to train on dataset five, compared to $\$ 0.337122$ USD for RandomSubspace. Based on escalating data, this cost would increase with each update and soon be significant. Similarly, the time taken to report a decision i.e. testing could be equally costly. With the onslaught of malware,

\footnotetext{
${ }^{1}$ https://aws.amazon.com/ec2/pricing/on-demand
} 
bottlenecks in training could lead to a build up of unused samples, affecting storage costs, training times and ultimately classification accuracy.

This work presents several novel contributions to the literature. First, 23 classifiers were investigated on the novel dataset, the most comprehensive investigation in the area to date. While all previous research has used fixed datasets, we present the performance of classifiers on datasets simulating real retraining costs which would be expected in an implementation of this work.

Second, we show that the top 16 classifiers showed very high accuracies, supporting the hypothesis that dynamic opcode analysis is a viable malware detection strategy.

Third, the high levels of accuracy achieved on default parameters demonstrated that complex and expensive tuning phases are not automatically required, minimising the risk of overfitting to the training data.

Forth, the research presented offers the first examination of the cost of retraining and testing updatable and nonupdatable classifiers, both parallelised and non-parallelised, with escalating malware datasets. It is shown that ensembled tree-based classifiers offer fast training and testing times, with high levels of accuracy and low retraining penalties. While criticism is sometimes levied at 'basic' classifiers, such as Random Forest, the research clearly demonstrates that for the current problem it was (i)fast to train; (ii) even faster to test; (iii) scalable; (iv) able to cope with data explosion; (v) robust and, most importantly (vi) highly accurate.

These key findings inform the current body of literature on the feasibility of the application and implementation of machine learning techniques in opcode-based malware detection. This will shape future research into how to actually apply these strategies in anti-malware systems, taking the work from academia to application.

\section{REFERENCES}

[1] S. Vemparala, F. D. Troia, V. A. Corrado, T. H. Austin, and M. Stamp, "Malware detection using dynamic birthmarks," in IWSPA 2016. Proceedings of the 2016 ACM International Workshop on Security and Privacy Analytics, co-located with CODASPY 2016, 2016, pp. 41-46.

[2] "Mcafee labs threats report june 2017," McAfee, Tech. Rep., 2017.

[3] P. Domingos and G. Hulten, "Mining high-speed data streams," in Proceedings of the sixth ACM SIGKDD international conference on Knowledge discovery and data mining. ACM, 2000, pp. 71-80.

[4] D. Carlin, A. Cowan, P. O'Kane, and S. Sezer, "The effects of traditional anti-virus labels on malware detection using dynamic runtime opcodes," IEEE Access, vol. 5, pp. 17742-17752, 2017.

[5] I. Santos, F. Brezo, B. Sanz, C. Laorden, and P. G. Bringas, "Using opcode sequences in single-class learning to detect unknown malware," IET information security, vol. 5, no. 4, pp. 220-227, 2011.

[6] N. Runwal, R. M. Low, and M. Stamp, "Opcode graph similarity and metamorphic detection," Journal in Computer Virology, vol. 8, no. 1-2, pp. 37-52, 2012.

[7] B. Anderson, D. Quist, J. Neil, C. Storlie, and T. Lane, "Graphbased malware detection using dynamic analysis," Journal in Computer Virology, vol. 7, no. 4, pp. 247-258, 2011.

[8] P. OKane, S. Sezer, K. McLaughlin, and E. G. Im, "Svm training phase reduction using dataset feature filtering for malware detection," IEEE transactions on information forensics and security, vol. 8, no. 3-4, pp. 500-509, 2013.

[9] D. Carlin, P. O'Kane, and S. Sezer, Dynamic Analysis of Malware using Run Time Opcodes, 1st ed., ser. Data Analytics and Decision Support for Cybersecurity - Trends, Methodologies and Applications. Springer, 2017.
[10] C. Rosset, "A review of online decision tree learning algorithms," Johns Hopkins University Department of Computer Science, Tech. Rep., 2015. [Online]. Available: http://corbyrosset.com/files/ OnlineDecisionTreeReview.pdf

[11] P. E. Utgoff, "Incremental induction of decision trees," Machine Learning, vol. 4, no. 2, pp. 161-186, 1989.

[12] P. E. Utgoff, N. C. Berkman, and J. A. Clouse, "Decision tree induction based on efficient tree restructuring," Machine Learning, vol. 29, no. 1, pp. 5-44, 1997.

[13] S. Lee and C.-H. Jun, "Fast incremental learning of logistic model tree using least angle regression," Expert Systems with Applications, vol. 97, pp. 137-145, 2018. [Online]. Available: https://www.scopus.com/inward/record.uri?eid=2-s2 $0-85038868023 \&$ doi $=10.1016 \% 2$ fj. eswa.2017.12.014\&partnerID $=40 \&$ md5=2a139e2180c01b27493a3620fe 122936

[14] T. Sethi and M. Kantardzic, "Handling adversarial concept drift in streaming data," Expert Systems with Applications, vol. 97, pp. 18-40, 2018. [Online]. Available: https://www.scopus.com/inward/ record.uri?eid $=2-\mathrm{s} 2.0-85038210723 \&$ doi $=10.1016 \% 2 \mathrm{fj}$.eswa.2017.12. 022\&partnerID $=40 \&$ md5 =fd4b3bd253bc25e87ac4104a599c72e5

[15] "An incremental associative classification algorithm used for malware detection," Proceedings of the 2010 2nd International Conference on Future Computer and Communication, ICFCC 2010, vol. 1, pp. V1757V1760, Jan-1 2010.

[16] K. Rieck, P. Trinius, C. Willems, and T. Holz, "Automatic analysis of malware behavior using machine learning," Journal of Computer Security, vol. 19, no. 4, pp. 639-668, 2011, cited By :172.

[17] N. A. Huynh, W. K. Ng, and K. Ariyapala, "A new adaptive learning algorithm and its application to online malware detection," in International Conference on Discovery Science. Springer, 2017, pp. 18-32.

[18] "Vxheaven," 2014. [Online]. Available: http://vxheaven.org/vl.php

[19] J.-M. Roberts, VirusShare.com, 2014

[20] A. Nappa, M. Z. Rafique, and J. Caballero, Driving in the cloud: An analysis of drive-by download operations and abuse reporting, ser. Detection of Intrusions and Malware, and Vulnerability Assessment. Springer, 2013, pp. 1-20.

[21] N. Chawla, N. Japkowicz, and A. Kolcz, "Special issue on class imbalances," SIGKDD Explorations, vol. 6, no. 1, pp. 1-6, 2004.

[22] K. Lejska, "X86 opcode and instruction reference." [Online]. Available: http://ref.x86asm.net/

[23] P. O'Kane, S. Sezer, K. McLaughlin, and E. G. Im, "Malware detection: program run length against detection rate," IET software, vol. 8, no. 1, pp. 42-51, 2014.

[24] D. Bilar, "Opcodes as predictor for malware," International Journal of Electronic Security and Digital Forensics, vol. 1, no. 2, pp. 156-168, 2007.

[25] B. Kang, K. S. Han, B. Kang, and E. G. Im, "Malware categorization using dynamic mnemonic frequency analysis with redundancy filtering," Digital Investigation, vol. 11, no. 4, pp. 323-335, 122014.

[26] E. Frank, "Weka mailing list," 2013.

[27] R. Kohavi, "Wrappers for performance enhancement and oblivious decision graphs," Ph.D. dissertation, 1995.

[28] I. H. Witten, E. Frank, M. A. Hall, and C. J. Pal, Data mining :practical machine learning tools and techniques, 4th ed. Cambridge, MA; 4: Morgan Kaufmann, 2017, ian H. Witten, Eibe Frank, Mark A. Hall, Christopher J. Pal.; xxxii, 621 pages :illustrations ;24 cm; texttxt; still imagesti; unmediatedn; volumenc; Previous edition: 2011.; Includes bibliographical references and index.

[29] L. Breiman, "Random forests," Machine Learning, vol. 45, no. 1, pp. 5-32, 2001.

[30] T. K. Ho, "The random subspace method for constructing decision forests," IEEE Transactions on Pattern Analysis and Machine Intelligence, vol. 20, no. 8, pp. 832-844, 1998.

[31] E. Frank and I. H. Witten, "Generating accurate rule sets without global optimization," 1998.

[32] L. Breiman, "Bagging predictors," Machine Learning, vol. 24, no. 2, pp. 123-140, 1996.

[33] D. W. Aha, D. Kibler, and M. K. Albert, "Instance-based learning algorithms," Machine Learning, vol. 6, no. 1, pp. 37-66, 1991.

[34] J. R. Quinlan, "C4. 5: Programming for machine learning," Morgan Kauffmann, vol. 38, 1993.

[35] W. W. Cohen, "Fast effective rule induction," in Proceedings of the twelfth international conference on machine learning, 1995, pp. 115123.

[36] N. Landwehr, M. Hall, and E. Frank, "Logistic model trees," Machine Learning, vol. 59, no. 1-2, pp. 161-205, 2005. 
[37] R. Kohavi, "The power of decision tables," Machine learning: ECML95, pp. 174-189, 1995.

[38] R. C. Holte, "Very simple classification rules perform well on most commonly used datasets," Machine Learning, vol. 11, no. 1, pp. 63-90, 1993.

[39] Y. Freund and R. E. Schapire, "Experiments with a new boosting algorithm," in Icml, vol. 96, 1996, pp. 148-156.

[40] W. Iba and P. Langley, "Induction of one-level decision trees," in Proceedings of the ninth international conference on machine learning, 1992, pp. 233-240.

[41] B. Schölkopf, C. J. Burges, and A. J. Smola, Advances in kernel methods: support vector learning. MIT press, 1999.

[42] G. Hulten, L. Spencer, and P. Domingos, "Mining time-changing data streams," in Proceedings of the seventh ACM SIGKDD international conference on Knowledge discovery and data mining. ACM, 2001, pp. 97-106.

\section{AUTHOR CONTRIBUTIONS}

Domhnall Carlin was the main author of this work, and conducted all experiments. All authors materially contributed to the experimental motivation, design, the analysis and interpretation of the data and the drafting of the paper. 


\section{APPENDIX A \\ DESCRIPTION OF CLASSIFIERS USED}

The following is a brief synopsis of the classification algorithms used, as explained by the WEKA developers in [28].

- RandomForest: Random Forest is an ensemble learner, which combines the decisions of multiple smaller learners (decision trees). RF differs from traditional tree-based learning algorithms, in that a random number of features are used at each node in the tree to decide the parameter, which improves noise immunity and reduces the tendency to overfit. See [29].

- RandomCommittee: An ensemble of randomizable base classifiers, each of which is built using a different random number seed. The overall output prediction is an average of the predictions outputted by the base classifiers.

- RandomSubspace: A ensemble of decision trees built by using pseudorandomised subsets of features (i.e. subspaces), which then combine to form the overall classifier. It maintains highest training accuracy and generalization accuracy increases with complexity growth. See [30].

- PART: The rule-based PART decision list generates a partial C4.5 tree at every iteration, taking the best leaf an making it into a rule. See [31].

- Bagging: Bootstrap aggregating is an ensemble method which attempts to reduce the bias-variance decomposition, the two sources of error in classification. The algorithm resamples the training data on each iteration, deleting and synthetically generating new instances, and training a classifier on each new set. A voting procedure then selects the class from the outputs of the weak learners. See [32].

- IBk:A KNN-based classifier, which is a type of lazy learner i.e. it has no traditional training phase, but classifies instances at testing time. See [33].

- J48: The WEKA implementation of the C4.5 decision tree algorithm. See [34].

- JRip: The WEKA implementation of the RIPPER rules-based classifier. See [35].

- RandomTree: A tree-based learner that considers $\mathrm{K}$ attributes, which are randomly chosen at each node.

- REPTree: A fast decision tree algorithm which constructs a decision tree using information gain, using reduced-error pruning with backfitting.

- LMT: Logistic Model Tree classifier i.e.trees which use logistic regression functions at the end nodes. See [36].

- DecisionTable: One of the simplest classification algorithms, Decision Table constructs a rules-based table for classifying new instances based on the training data. See [37].

- OneR:A OneRule classifier, which generates one rule for each predicting attribute, selecting the lowest error attribute as its single rule. See [38].

- AdaBoostM1:An adaptive boosting algorithm which combines the output of its component learners into a weighted sum output, and adapts subsequent learners to misclassified instances. See [39].

- DecisionStump:A one-level decision tree with only one internal node (i.e. root) which connects to the terminal nodes (i.e. leaves). [40].

- SGD:Stochastic Gradient Descent for various implemented classifiers (SVM in the current work).

- SMO: Implements the Sequential Minimal Optimization algorithm for training an SVM. [41].

- HoeffdingTree:An implementation of the VFDT, which is an incremental decision tree, capable of learning from massive streaming datasets. [3], [42].

- ZeroR:WEKA implementation of the 0-R rule-based learner.

- MultilayerPerceptron:A feed-forward artificial neural network classifier that uses backpropagation to classify instances.

\section{APPENDIX B}

\section{PERFORMANCE METRICS}

- Number of training instances Number of samples used for training the model.

- Number of testing instances Number of samples used for testing the model.

- Number correct Number of correct classifications.

- Percent correct Number of correct predictions, of both classes, out of all classifications made. Also referred to as accuracy, i.e. the percentage of correctly assigned classes (0\% indicating a poor classifier, 100\% showing perfect accuracy).

$$
A C C=\frac{\mathrm{TP}+\mathrm{TN}}{\text { TotalPopulation }}
$$

- Number incorrect Number of incorrect classifications, of both classes

- Percent incorrect Number incorrect out of all classifications made

- Percent unclassified Number of unclassified out of all classifications made

- Number unclassified Number of classifications which could not be made

- Kappa statistic Compares the observed accuracy in the context of an expected accuracy i.e. random chance, providing more information about the performance of the classifier. For example, an observed accuracy of $90 \%$ can be viewed as 
performing better when the expected accuracy is $50 \%$ than $80 \%$. This can be seen in the formula: $K=\frac{O-E}{1-E}$, where $O$ is observed accuracy and $E$ is expected accuracy.

- Number true positives Number of true positive detections out of all positive samples.

- True positive rate (TPR)/Recall/Sensitivity True positive out of all that were positive.

- Number false positives/False positive rate (FPR)

Falsely classified as positive out of all that were truly negative.

- Number true negatives

Number classified as negative when they were truly negative

- Number false negatives/False negative rate (FNR)

False negative out of actually positive

- Precision/Positive Predictive Value True positive class out of predicted positive class

- True negative rate (TNR)/Specificity True negative out of actually negative

- $\boldsymbol{F} / \boldsymbol{F}_{1}$ measure/score $F_{1}$ score is the harmonic mean of precision and sensitivity (1 is the ideal), given by the formula

$$
F_{1}=\frac{2 \mathrm{TP}}{2 \mathrm{TP}+\mathrm{FP}+\mathrm{FN}}
$$

It is used as a more complex measure of a classifier's accuracy, beyond simply correctly classified instances. It is worth noting that the F-measure does not take into account the true negative classifications, which may be viewed as a disadvantage when compared to Kappa or MCC.

- Matthews correlation coefficient (MCC) A correlation coefficient between the predicted and observed classifications. MCC is used as a measurement of the quality of a binary classifier, and is particularly useful with imbalanced classes, as it accounts for all false/positive measures:

$$
M C C=\frac{T P \times T N-F P \times F N}{\sqrt{(T P+F P)(T P+F N)(T N+F P)(T N+F N)}}
$$

\section{- AUROC}

Area Under Receiver Operating Characteristic curve, i.e. when the True Positive \% rate is plotted against the False Positive $\%$ rate, the area underneath this curve ( 1 is the ideal).

- AUPRC Area Under Precision Recall curve (1 is the ideal) plotted with precision against recall, where

$$
P R C=\frac{\text { Precision }=\frac{T P}{T P+F P}}{\text { Recall }=\frac{T P}{T P+F N}}
$$

